NASA Technical Memorandum 88319

\title{
A Numerical Study of Incompressible Juncture Flows.
}

Dochan Kwak, Stuart E. Rogers, Upender K. Kaul, and James L. C.Chang 


\section{A Numerical Study of Incompressible Juncture Flows}

Dochan Kwak, Ames Research Center, Moffett Field, California Stuart E. Rogers,

Upender K. Kaul, Sterling Software, Palo Alto, California

James L. C. Chang, Rocketdyne, Rockwell International, Canoga Park, California

August 1986

\section{N/Sก}

National Aeronautics and

Space Administration 


\section{SUMMARY}

The laminar. steady juncture flow around single or multiple posts mounted between two flat plates is simulated using the three-dimensional incompressible Navier-Stokes code, INS3D. The three-dimensional separation of the boundary layer and subsequent formation and development of the horeshoe vortex is computed. The computed flow compares favorably with the experimental observation. This report summarizes the recent numerical study performed at NASA Ames Research Center to understand and quantify the juncture flow relevant to the Space Shuttle main engine power head.

\section{INTRODUCTION}

This study analyzes the local flow around liquid oxygen (LOX) posts in the Space Shuttle main engine (SSME). As shown schematically in figure 1, LOX posts are densely packed in the main injector assembly into which very nonuniform hot gas flows from the transfer ducts (ref. 1). As a first step toward a complete flow analysis around the LOX post, This study deals with idealized cases of laminar, steady juncture flows around a single post and single and double rows of posts mounted between two flat plates.

The flow around a cylinder-plate juncture produces a very interesting viscous phenomenon caused by the interaction between the boundary layer from the plate and the cylinder. The three-dimensional (3-D) separation of the boundary layer and subsequent formation of the socalled horseshoe vortex and its development is very challenging to analyze both experimentally and numerically. This type of flow can occur in many practical engineering problems. Flow around a wing-fuselage junction is a simple example of its kind and the flow near the end wall of turbomachinery blades might be one of the most complicated problems. Most of the earlier studies on juncture flow have been experimental. Baker (ref. 2) shows that laminar juncture flow is confined to a very limited region. A similar result has been obtained most recently by Thomas (ref. 3). Eckerle and Langston (ref. 4) reported a single primary vortex and saddle point contrary to multiple vortex systems observed earlier by other researchers. Interpretation of the phenomenon also varies (refs. 3 and 5 ).

Computational simulation of these flows involves distinctively different features from that of external aerodynamics. For instance, the thickness of viscous layer for these types of flows is of the same order as the characteristic flow-field dimension, while the viscous region tends to be confined in a thin layer near the body for external flows. Realistic juncture flows under an internal flow enviroment are likely to have large deflection as in the case of LOX post regions in the SSMF. Separated and recirculating zones need to be resolved in the wake region of the first and second rows of posts in the two-row cases. Recently, some numerical studes on this flow have been attempted. Kaul et al. (ref. 6) reported an extensive numerical study on a single cylinder-plate flow. Subsequently, Rogers et al. (ref. 7) simulated flow around a multiple-post arrangement. Independently, Kiehm et al. (ref. 8) reported a numerical study of flow around a single post in a channel. These computations reported qualitatively similar phenomena. This report summarizes the recent numerical study performed at NASA Ames Research Center to understand and to quantify the laminar juncture flow relevant to the SSME-type flow. The turbulence modeling of the juncture flows will constitute the second phase of the study.

Special thanks to Dr. G. Schewe of DFVLR, West Germany for providing his oil-flow visualization picture. This work is partially sponsored by NASA Marshall Space Flight Center. 


\section{SOLUTION PROCEDURE}

Since the Mach number of the flow is less than 0.12 (ref. 1), the flow field is computed using the incompressible Navier-Stokes flow solver, INS3D (ref. 9), which utilizes an implicit finite difference procedure in generalized curvilinear coordinates. This code has been verified for both two-dimensional (2-D) and 3-D test problems, and has been applied to several realistic problems in acrospace design. Most notably, the application to the SSME flow-field analysis has made a significant impact on the Shuttle program.

This code was used as a test code for the Numerical Aerodynamic Simulator (NAS) Facility Cray 2 at NASA Ames Research Center. Most of the numerical results presented here were obtained on the Cray 2.

\section{RESULTS}

Figure 2 shows the computational domain for a single post on a flat plate. This is an extreme idealization of the flow around a post in the multiple-post assembly in figure 1. However, an understanding of this flow is of considerable value toward a full analysis of the entire assembly. Upstream boundary layer thickness is varied by using partially and fully developed channel-flow profiles. The convergence characteristics of the flow solver are shown in figure 3 by the history of RMSDQ, which denotes the root-mean-square value of the change per iteration in the pressure and velocities. The three curves in the figure show three variations of the INS3D code; namely a block tridiagonal, a diagonal, and a diagonal version with fourth order implicit smoothing terms. The flow solver converges rapidly to about four orders of magnitude reduction in RMSDQ. The computing time per iteration per grid point is $91 \mu \mathrm{sec}$ for the block tridiagonal version and 32 $\mu$ sec for the diagonal version of the code. Figure 4 shows the particle traces for a single post at $\operatorname{Re}=1000$. The saddle point separation and horseshoe vortex can be seen from the traces near the flat plate. The secondary flow in front of the cylinder wraps around toward the wake region and forms a counter-rotating pair of vortex filaments. These spiraling twin vortices demonstrate a striking difference between this type of juncture flow and a 2-D cylinder. The vortex filaments are washed upward and then attenuate as they interact and move downstream. In reality, vortex shedding and possible unsteady motion take place at this stage. These "tornado-shaped" vortices are very difficult to observe experimentally, and validation of this phenomenon was very much needed. Recently, Schewe (Schewe, G., Private communication. 1985. DFVLR, West Germany.) made oil-flow visualization around a single post, showing clear evidence of the twin vortex behind the cylinder (fig. 5). This experimental observation is qualitatively similar to the computed results. shown by the particle traces in figure 4 and the isobars near the flat plate in figure 6 . This juncture flow structure will lead to a strong variation in skin friction and pressure along the cylinder and hence significantly affects the overall loading on the post.

Figures 7 through 10 show the computational results for a multiple-post arrangement. The horizontal plane of the C-H-type hybrid grid which used for two-row computation is shown in figure 7 . This $2-D$ grid is stacked up vertically to form a 3-D grid. A total of 120,000 grid points were used for the calculation on the Cray-XMP. For the computation on the Cray 2 , a refined grid with 463,000 mesh points was used. In the y-direction, periodicity is assumed. Comparison for the mass averaged total pressure drop between the single row and the double row of posts is shown in figure 8 . In this figure, the first and second rows of the two-row post arrangement are designated as double row 1 and 2 , respectively. The varying incidence angle corresponds to various relative 
locations of the posts in figure 1(b). Zero angle corresponds to the centerline of the center transfer duct and the angle increases clockwise. The angular dependency shown for the single row case is partially confirmed computationally by Chang and Yang (will be published as a continuation of reference 1). Figure 9 shows the particle traces near the post plate junction for two rows of posts. Twin vortices behind the first row of posts are still observed and the saddle point of separation for the second row of posts is influenced by the presence of the spiraling vortices behind the first row as shown in figure 10 (a). Whether, in reality, the flow maintains steadiness behind the second row at this Reynolds number remains to be verified. The present flow solver is designed for obtaining steady-state solutions, and the results can be regarded as an ensemble average in the wake region of the second row of posts. Figure 10 (b) shows another tornado-type vortex generated behind the second row when the incident angle is increased to $78^{\circ}$. Understanding the vortex dynamics and quantifying the resulting load on posts will be of significant engineering importance as well as an important contribution to the basic understanding the flow physics of juncture flows.

\section{CONCLUDING REMARKS}

The present study shows the computational capability to study the juncture flow relevant to the SSME LOX posts. The computed flow compares favorably with experimental observation. By comparing the multiple-row post loading with the single-row post loading and varying the angles of flow incidence, the degree of interaction between the front and the rear rows of posts can be quantitatively determined. An improved multiple-row post arrangement therefore can be determined based on the results from the present study. High Reynolds number flows need to be simulated to analyze more realistic cases in the SSME. This will require that special attention be given to the turbulence models for an accurate prediction of the loading on the posts. To complete the juncture flow study, a time-dependent flow solver will be essential. 


\section{REFERENCES}

1. Chang. J. L. C.; Yang, R-J; and Kwak, D.: A Full Navier-Stokes Simulation of Complex Internal Flows. Tenth Intern. Conf. Numerical Methods in Fluid Dynamics, Beijing, Peoples Republic of China, July 1986.

2. Baker, C. J.: The Laminar Horseshoe Vortex. J. Fluid Mech., vol. 95, part 2, June 1979, pp $346-367$.

3. Thomas, A.: Laminar Juncture Flow - A Visualization Study. Lockheed-Georgia Co., (in press 1986).

4. Eckerle, W. A. and Langston, L. S.: Horseshoe Vortex around a Cylinder. ASME International Gas Turbine Conference, Dusseldorf, West Germany, June 1986.

5. Peake, D. J. and Tobak, M.: Three-Dimensional Interactions and Vortical Flows with Emphasis on High Speeds. NASA TM 81169, 1980.

6. Kaul, U. K.; Kwak, D.; and Wagner, C.: A Computational Study of Saddle Point Separation and Horseshoe Vortex System. AIAA Paper 85-0182, Jan. 1985.

7. Rogers, S. E.; Kaul, U.; and Kwak, D.: A Numerical Study of Single and Multiple LOX Posts and lts Application to the Space Shuttle Main Engine. AIAA Paper 86-0353, Jan. 1986.

8. Kiehm. P.; Mitra, N. K.; and Fiebig, M.: Numerical Investigation of Two- and ThreeDimensional Confined Wakes behind a Circular Cylinder in a Channel. AIAA paper 86-0035, Jan. 1986.

9. Kwak. D.; Chang, J. L. C.; Shanks, S. P.; and Chakravarthy, S.: A Three-Dimensional Incompressible Navier-Stokes Flow Solver Using Primitive Variables, AlAA J, vol. 24, no. 3. Mar. 1986, pp 390-396. 


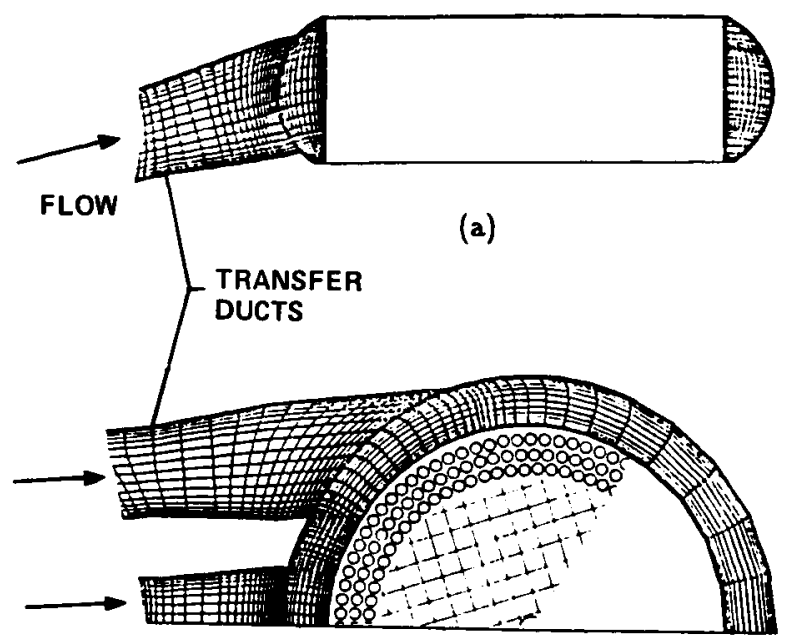

(b)

Figure 1 Schematic of the LOX posts in the SSME.

(a) Vertical cross section.

(b) Horizontal cross section.

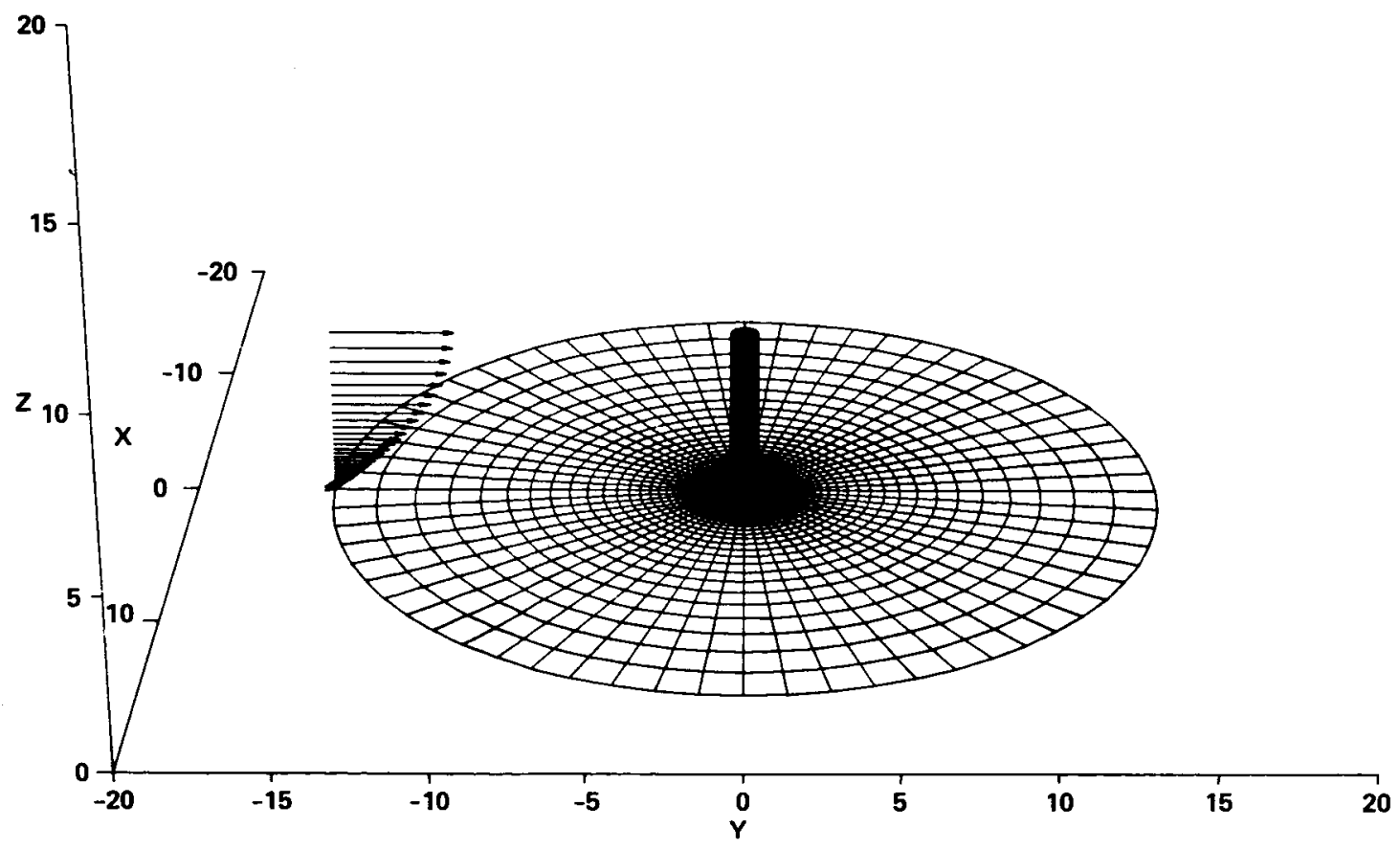

Figure 2 Grid for a single post. 


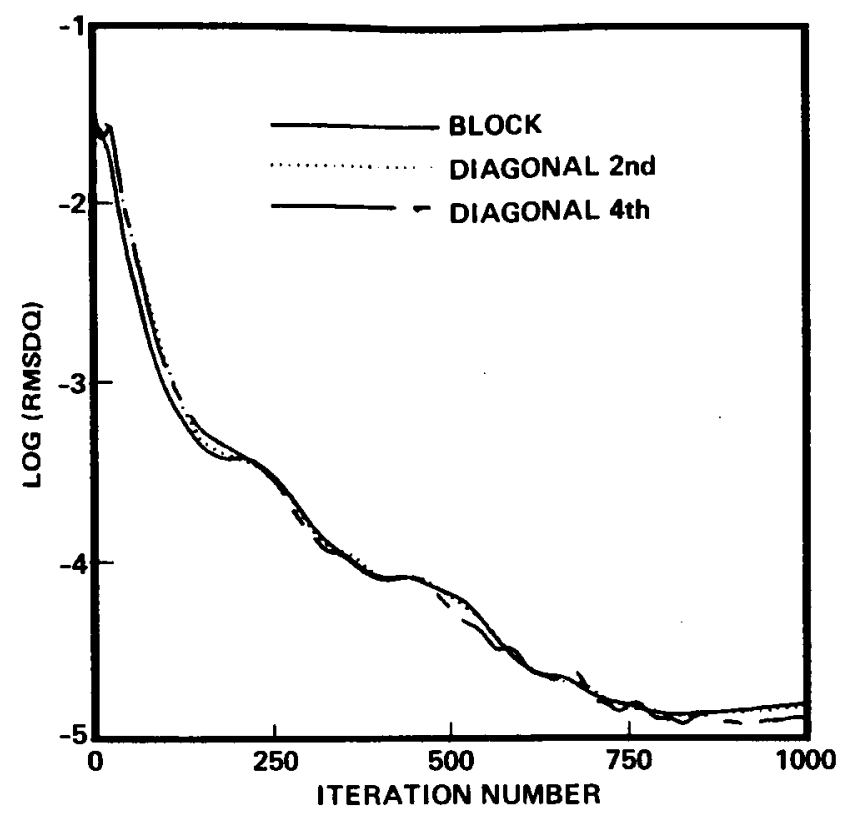

Figure 3 Convergence history for a single post.

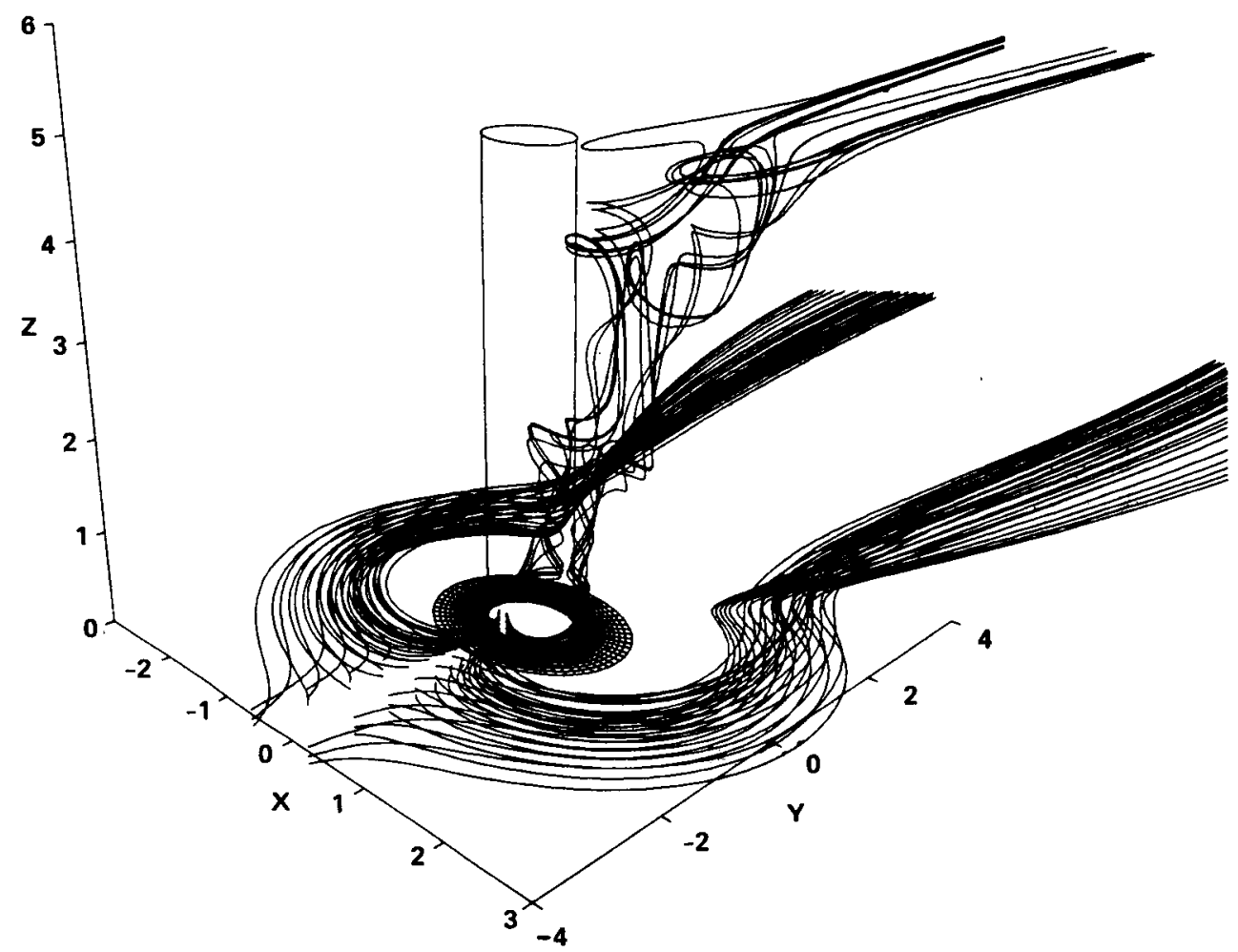

Figure 4 Particle traces for a single post at $R e=1000$. 


\section{ORIGINAL PAGE IS
OF POOR QUAITY}

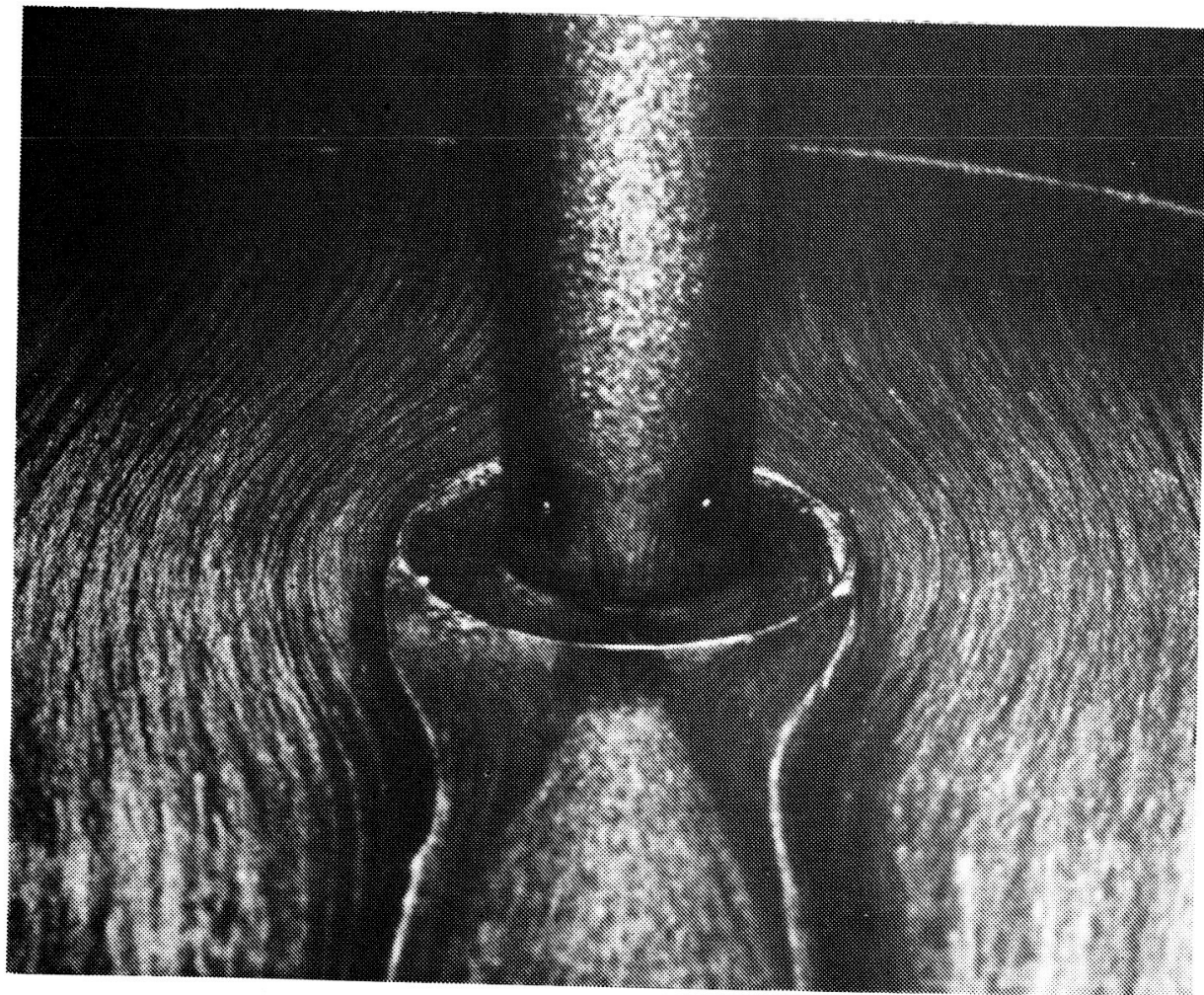

Figure 5 Flow around a single post at $\operatorname{Re}=1.85 \times 10^{5}$ (oilflow visualization by $\mathrm{G}$. Schewe.)

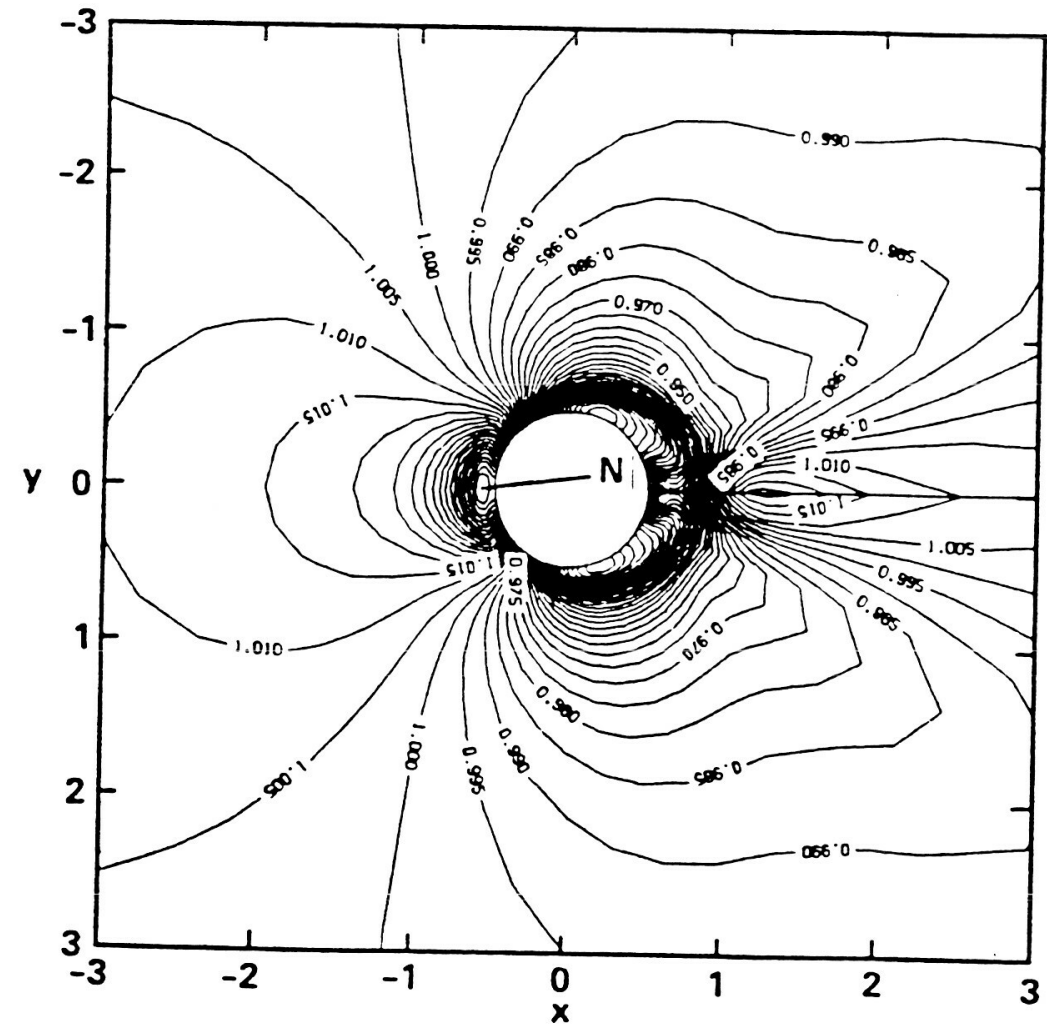

Figure 6 Isobars in the horizontal plane at $\mathrm{z} / \mathrm{D}=0.01$. 


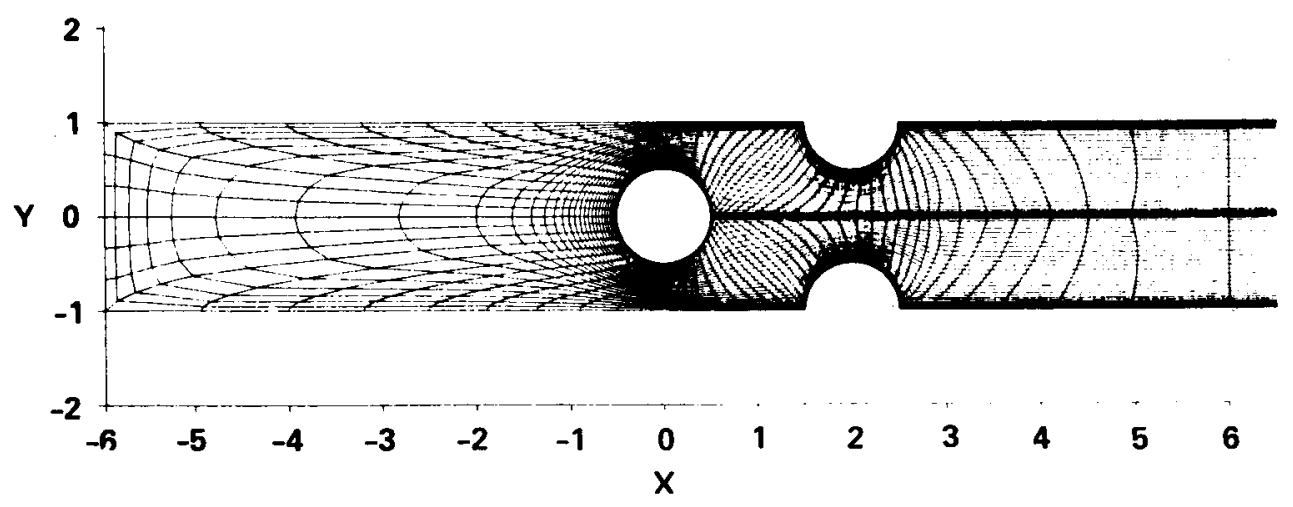

Figure 7 Grid for two rows of posts.

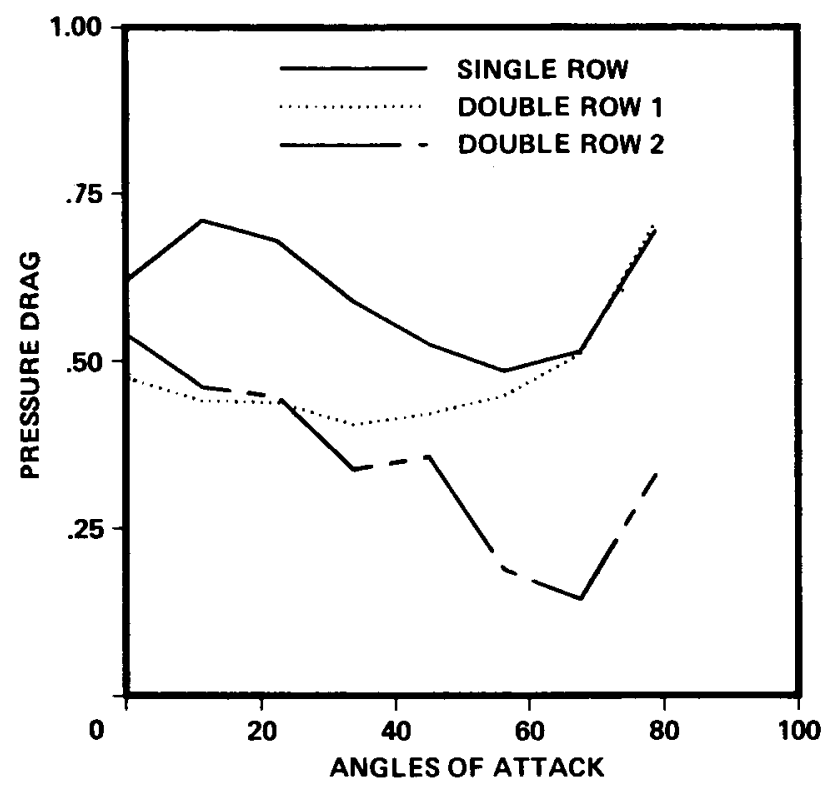

Figure 8 Total pressure drop per unit of mass flow versus angle of attack, $\mathrm{Re}=1000$.

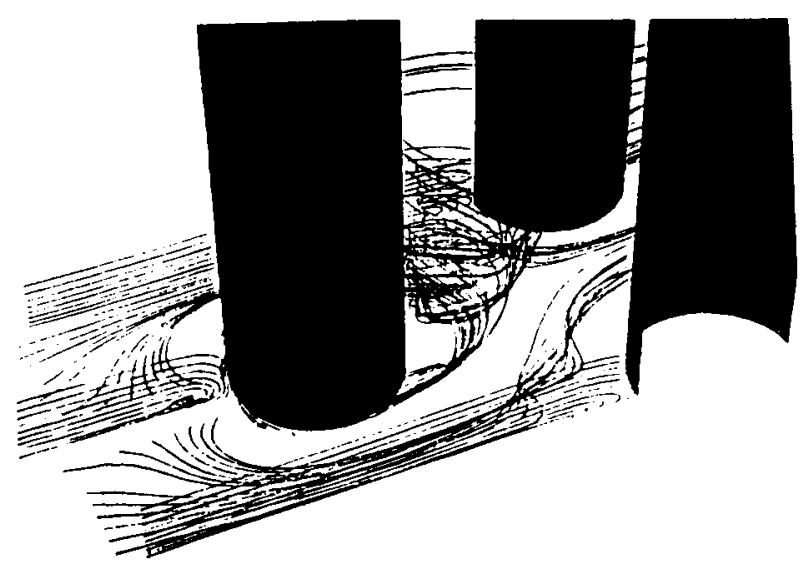

Figure 9 Particle traces neàr the post-plate junction for two rows of posts. 


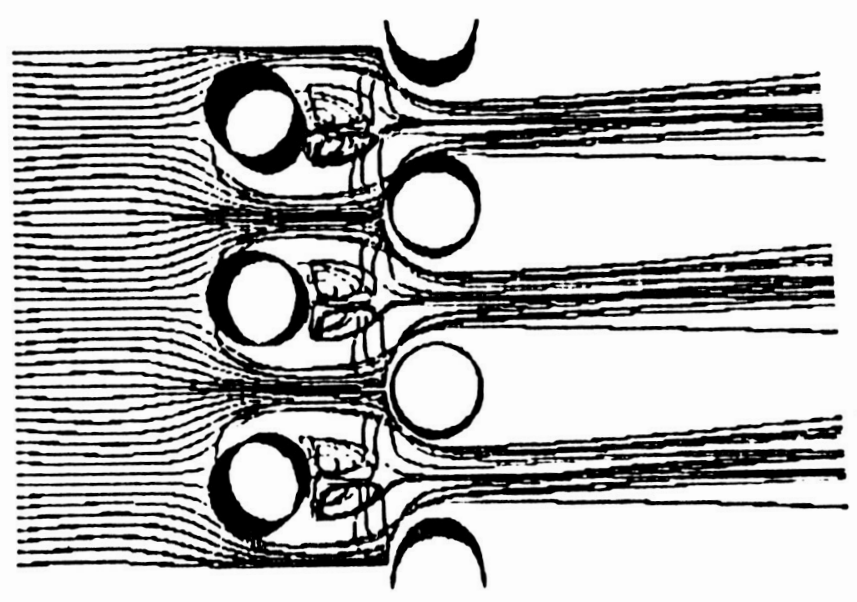

(a)

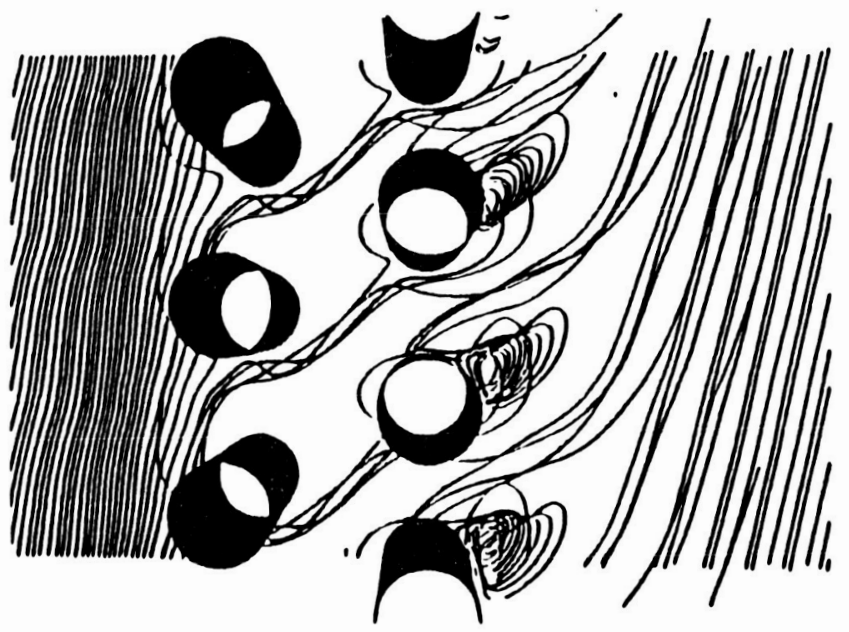

(b)

Figure 10 Particle traces for two rows of postsat $\operatorname{Re}=1000$ :

(a) $0^{\circ}$ incidence, (b) $78^{\circ}$ incidence. 


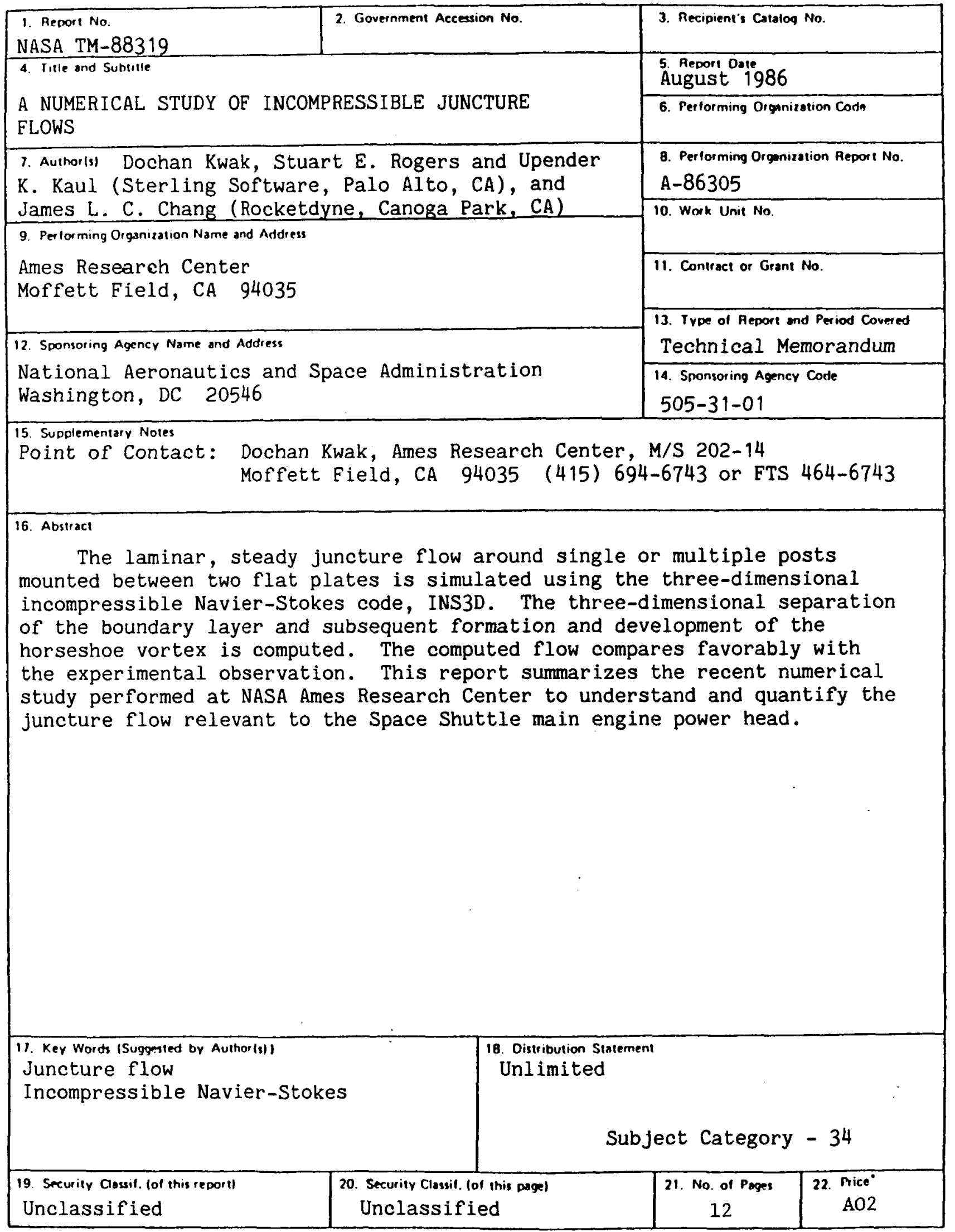

"For sate by the National Technical Information Service, Springfield, Virginia 22161 